\title{
Open Dressing in Management of Second Degree Burn in a Pediatric Burn unit
}

\author{
M S Ullah ${ }^{1}$, K M N U Ferdous ${ }^{2}$, S Ara ${ }^{3}$, J Bajracharia ${ }^{4}$
}

\section{Abstract}

This study was to evaluate the results of open dressing in management of second degree burn in children.

Methods : A retrospective study conducted on children with second degree burn during the period of 42 months from January 2010 to June 2014.

Results : 150 patients included in this study. Age range was 1 months 12 days to 11 years 10 months. $68 \%$ patients were within $1-5$ years. $70 \%$ injured by hot water, $16 \%$ by hot liquids, $8.7 \%$ by hot cooking related substance and $5.3 \%$ due to flame. Only $12 \%$ patients showed positive culture of pathogenic organisms. Among them, 4 had a combined growth of Pseudomonas auroginosa and E.coli. 2 (1.3\%) patient needed skin grafting. $62 \%$ patient stayed in the hospital up to 10 days and only 1 patient stayed 35 days. Mean cost of management per patient was calculated under several categories.

Conclusion : Second degree burn can manage by open method effectively and successfully. Regular cleansing of the burnt wound with clean water and application of moist exposed burn ointment (MEBO) can easily be tough to the attendants.

1 Dr.Md. Saif Ullah MS (Paediatric Surgery) Assistant Professor Dept. of Paediatric Surgery Dhaka Shishu Hospital (DSH) \& BICH

2 Dr. Kazi Md. Noor-ul Ferdous MS (Paediatric Surgery) Registrar Dept. of Paediatric Surgery Dhaka Shishu Hospital \& BICH

3 Dr. Sawkat Ara MBBS, MPH, USAID Fellow RMO

Dept. of Paediatric Surgery

Dhaka Shishu Hospital

4 Jasmine Bajracharia MS Student (Paediatric Surgery) Dept. of Paediatric Surgery Bangladesh Institute of Child Health (BICH)

\section{Correspondence}

Dr. Kazi Md. Noor-ul Ferdous MS (Paediatric Surgery) Registrar Dept. of Paediatric Surgery Dhaka Shishu Hospital \& BICH e-mail: kmnferdous@gmail.com
Key wards : Second degree burn, Open dressing, Pediatric.

\section{Introduction}

In the developing countries about $45 \%$ patients are children admitted in burn unit. It is said that burn is one of the most devastating causes of social, functional and psychological impairments in the whole world. $^{1}$

In 2003, a population based survey reported, burn was the fifth leading cause of child health illness among the children of 1-17 years age group in Bangladesh. The overall incidence of burn was 288.1 per 100,000 children per year and the morbidity was 0.6 per 100,000 children of the same age group. ${ }^{2}$

Total 23,000 people died due to burn in the year 2000 around the whole world. Among them the majority occurred in low and middle income countries. ${ }^{2}$ Burn injury was the second most common surgical problem in pediatric age group in the department of Pediatric surgery, Dhaka Shishu (Children) Hospital. ${ }^{3}$

In the management strategy of a burn patient, one must first assess the overall physiologic status of the child as with trauma related injury, the primary and secondary survey are performed in accordance Trauma Life Support standered. ${ }^{4}$ After the primary assessment a burn specific secondary survey should be performed that include a detailed assessment of the burn wounds and others. ${ }^{5}$

Traditional burn wound management involved applying topical antibiotic in dressing until the escher separated. ${ }^{6}$ Practically, the burn wound is better treated with dressing. Small children tend to prefer injured site to be covered with dressings. ${ }^{7}$ There are many reported methods regarding management of the burn wound. Open or exposed, semi-closed or closed technique. With or with out topical antibiotics , has all been advocated. The aim of open methods aims is drying up the burnt area as quick as possible, leaving tissues to heal under a dry crust. ${ }^{8}$

The open dressing reduces healing time and reepithelialization time and promotes early elimination of crusts. However, open dressing make it more difficult for patient with large burned areas to move and the occlusive dressing allows for the patient to move freely. ${ }^{9}$

Total management cost of burned patients is very high as it depends on lengthy hospital stay and demands multidisciplinary teams. Local care of burn wounds continues to account for a large proportion of the cost per day for treating patients with burns. Dressing costs, however, can vary according to the size and depth of the burned area, the type of dressing used and the occurrence of complications. ${ }^{9}$

Many believe the closed technique is ideal for management of second degree burn wounds. But Gosselin et al found the open or exposure technique is better for the resource-poor, low-income developing countries, in terms of reduced morbidity, length of hospital stay and treatment cost. ${ }^{8}$

Despite, a developing country with limited resourced, every year we are spending huge amount of money, working hours and man power for the management of burn wounds and their complications.

\section{Materials and Methods}

This retrospective study was conducted during the period of 42 months from January 2010 to June 2014, in the burn \& plastic, reconstructive unit of the department of pediatric surgery, Dhaka Shishu (children) Hospital.

Patients aged between with 0-12 years with second degree superficial burn, $>10 \%$ TBSA (total burn surface area) with involving any region of the body and any \% TBSA involving the hands, feet, face and perineum admitted with second degree burn and whom treated by exposed or without closed dressing were included in this study.

Patients arrived more than 24 hours after burn, burn with other systemic illness e.g.-Protein Energy 
Malnutrition, Cerebral Palsy, Myopathy, Neurological disorders, etc were excluded.

We included and evaluate only those patients from the hospital records, whom were managed by same protocol of burn management.

Prophylactic intravenous broad spectrum antibiotics (Inj. Cephradine, Inj. Flucloxacillin and Inj. Amikacin) were given. Initial analgesia was provided by injectable narcotic analgesic (Pethidine), then per rectal Diclofenac Sodium and oral Paracetamol. Oral Promethazine $\mathrm{HCl}$ and oral $\mathrm{H}_{2}$ blocker (Ranitidine) was also given.

The burn surface area initially cleaned with normal saline $(0.9 \% \mathrm{NaCl})$, then $2 \%$ Silver Sulphadiazine was applied keeping the wound uncovered or exposed. Patients were instructed to take daily bath using warm water followed by cleansing of the burn surface by normal saline and application of $2 \%$ Silver Sulphadiazine cream again.

Close follow up and inspection of the wound was done and any signs of wound infection (discharge, foul smell, pyrexia) were recorded. Wound swab was taken on the $3^{\text {rd }}$ day after admission for culture $\&$ sensitivity test.

If there was presence of purulent exudates, persistent pyrexia or devitalized tissue could not be removed by above mentioned procedure, decision of doing any surgical procedure was taken.

Patients were discharged when wound healed up without any pyrexia and no need for further procedure.

The following records were evaluated- evidence of bacterial wound infection, any operative procedure under general anesthesia, total number of days stayed in the hospital, and average cost of treatment per patient which includes admission fees, bed rent, operation charges, cost of various pathological investigations, cost to purchase intravenous fluids, normal saline, antiseptic cream, drugs, other dressing related materials etc.

\section{Results}

During the study period of 42 months, the admitted burn patients was about $6 \%$ of total pediatric surgery admission at Dhaka Shishu (children) Hospital. Out of them 150 patients with second degree superficial burn (scald) included in this study.

The lowest age of the patient of this study was 1 months 12 days and the highest age was 11 years 10 months. The age distribution is shown Figure- 1.

Among the study population, $103(68.7 \%)$ patient were male and $47(31.3 \%)$ patient were female.

Hot water, hot liquids like hot tea, hot milk, hot dal, hot rice water etc and flame were the main burning agents. Highest, $105(70 \%)$ by hot water and $8(5.3 \%)$ due to flame. Table-1

Out of 150 , only $18(12 \%)$ patients showed positive culture of pathogenic organisms. Among four of them had a combined growth of Pseudomonas auroginosa and E.coli. (Table-II) Culture sensitivity reports reveled that Staphylococcus aureus was sensitive to Flucloxacillin, Cephradin and Cftriaxone antibiotic. Pseudomonas was sensitive to Gentamycin, Ceftazidime And E.coli was sensitive to Gentamycin and Imipenum antibiotics. Infection was treated by using these sensitive antibiotics.
Figure 1 : distribution of age of the patient $(\mathrm{N}=150)$

\section{Age Distribution}

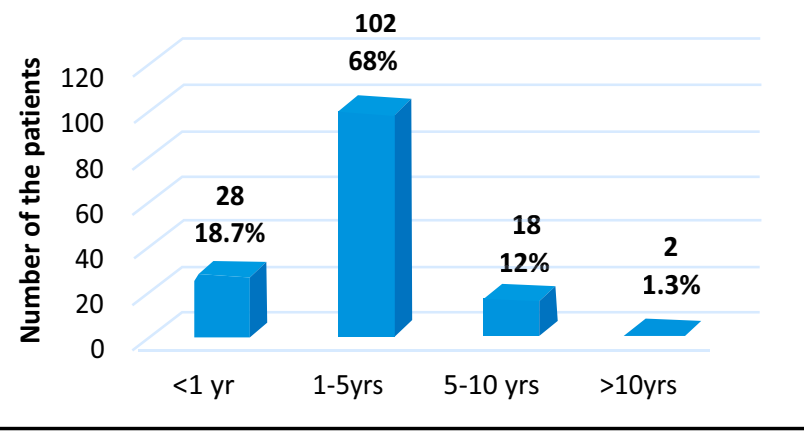

Table I : Burning Agents $(\mathrm{N}=105)$

Burning agents

Hot water

Number of patients

Percentage

Hot liquids

Hot cooking related substance

Flame

$\begin{array}{cc}105 & 70 \% \\ 24 & 16 \% \\ 13 & 8.7 \% \\ 8 & 5.3 \%\end{array}$

Table II : Wound infective Agents $(\mathrm{N}=18)$

Microorganisms

Number of patients

Percentage

Staphylococcal aureus

$10 \quad 60 \%$

E. Coli

4

$20 \%$

Pseudomonas auroginosa \& E.coli.

4

$20 \%$

Twenty (13.3\%) patients needed wound cleansing or debridement under general anesthesia only two to five time each. But only $2(1.3 \%)$ patients of them needed skin grafting.

Most of the patients (62\%) stayed in the hospital up to 10 days and only 1 patient stayed 35 days. Table- 3 .

\section{Table III : Length of hospital stay $(\mathrm{N}=105)$}

Days

Number of patients Percentage

Upto 10 days

93

$62 \%$

11 to 20 days

51

21-30 days

$>30$ days

5

$34 \%$

$3.3 \%$

$0.7 \%$

Mean costs of management per patient (whom did not received any operative procedures under general anesthesia) were calculated and shown in table 1 . Twenty patients needed operative procedures under general anesthesia like wound cleansing, debridement and skin grafting. Their management cost was a bit higher then others (Mean, 5500 Taka).

Table IV : Average costs of management per patient ( $N=130)$

Category of cost per patients

Taka

Admission fee \& bed rent

3915.65

Investigations

1520.60

Drugs

4280.45

Dressing \& other associate materials

950.50

Total

$10,667.20$ 


\section{Discussion}

After initial Advanced Trauma Life Support (ATLS) management to save the burnt patients life, the next issue is to reduce the possible immediate early morbidity by prompt resuscitation according to initial planned management protocol of burn wound management. Various techniques are available for burnt wound management. Still today there is no single technique is universally applicable for all types of burnt wound.

Common causative agents of burn in pediatric age group are hot water, hot liquids (tea, milk, rice water etc.), hot vegetable/curry, and flame. ${ }^{10}$ The repots are similar to our study, which showed most common agent was hot water and least common was flame burn.

Wound infection rate was significantly lower in this study. Three types of microorganisms were identified for the wound infection. Gosselin et al reported significant reduce wound infection rate in patients treated with open technique, which is similar to our present study. ${ }^{8}$ However, they did not mention the specific type of microorganisms isolated on culture in their study. In a previous study at same institute showed the same report and infection rate was lower in open dressing method in comparison to closed dressing. ${ }^{10}$

We managed all those patients by open dressing method. Demling RH et al mentioned, second degree superficial burn patients need 3-5 dressing in every 1-2 days interval ${ }^{11}$. As, in the close method, burn dressing should change regularly, it is very difficult to maintain in a developing country. In the other hand, in open method the burn unit worker or even the patents can apply local ointment after bathing the patient and clean the wound daily. More over, in some particular areas of the body like perineum, upper part of the thigh, glutial region close dressing is difficult, where open or exposed method is feasible.

To reduce the pain of the patient and to decrease psychological and physiological trauma, burn dressing should always be done under general anesthesia. ${ }^{12}$ We did all the dressing, which included wound cleansing, debridement under general anesthesia.

We observed that most of the studied patients stayed in the hospital less than ten days. This is similar to the study of Atityeh et al ${ }^{6}$. Hospital stay depends on other factors also like depth of burn, wound infection, Percentage of total burn surface area (\% TBSA), number of operative procedures. Our results of open method are similar to the study of Gosselin et al. ${ }^{8}$ The result also same to the study at same hospital, that showed length of hospital stay was less in open method. ${ }^{10}$
Most of the cases in this study did not need general anesthesia for wound care, thus mean management cost was less in those. The cost was increased when procedures done under general anesthesia. In the previous study at same hospital showed the cost of burn care was more in closed method than open dressing method. ${ }^{10}$ The cost of open dressing in this is same to that of previous study. However, the cost is depends on the economic status of the state.

\section{Conclusion}

Second degree burn can manage by open method effectively and successfully. Regular cleansing of the burnt wound with clean water and application of moist exposed burn ointment (MEBO) can easily be tough to the attendants.

\section{References}

1. Lawrence JC. Burns and scalds; etiology and prevention. In: Settle Ad, ed. Principles and practice of burn management, $1^{\text {st }}$ ed. Edinburgh: Churchill Livingstone, (1996), 313.

2. Mashreky SR, Rahman A, Chowdhury SM, Giashuddin S, Svanstrom L, Linan M, Shafinaz $\mathrm{S}$, Uhaa IJ, Rahman F. Consequences of childhood burn: Findings from the largest community-based injury survey in Bangladesh. Burns, (2008) 34, 912-918

3. Masood AFM, Khan AR, Islam KM. Surgical problems I children- 10 years survey in Dhaka Shishu (Children) Hospital. Dhaka Shishu (Children) Hospital Journal, (1991),7, 24-29.

4. Duffy BJ, McLaughin PM, Eichelberger MR. Assesment, Triage, and Early Management of Burns in Children', Clinical Pediatric Emergency Medicine, (2006), 7, 82-93

5. Shukla PC, Sheridan RL. Initial evaluation and management of burn patients. (2003), EMedicine.com Available at: www. Emedicine.com

6. Atiyeh BS, Dham R, Kadry M, Abdullah AF, Al-Oteify M, Fathi O, and Samir A. Benefitcost analysis of moist exposed burn ointment. Burns, (2002), 28, 659-669.

7. Carolyn R. Pediatric Burn. In: Settle Ad, ed. Principles and practice of burn management, $1^{\text {st }}$ ed. Edinburgh: Churchill Livingstone, (1996), 377-379.

8. Gosselin RA, Kuppers B. Open versus closed management of burn wounds in a lowincome developing country' Burns,(2008), 34, 644-647.

9. Torrati FG, Rossi LA, Ferreira E, Dalri MCB, Carvalho EC and Barberia CBS. Analysis of cost of dressings in the care of burn patients', Burns, (2000), 26, 289-291.

10. Saif Ullah M, Ferdous KMN, Haider MM, Sarwar MKA, Alam MR and Khan AR. Management of pediatric second-degree burn in a developing country: With or without close dressing?, Chattagram Maa-O-Shishu Hospital Medical College Journal, (2013), $12(2), 35-40$.

11. Demling RH, Way LW. 1994, 'Burn and other thermal injuries' in : Current surgical Diagnosis and Treatment. Way LW (ed). $10^{\text {th }}$ edn. Norwalk:Lange Medical Book, (1994), 241-56.

12. Simons MA, Kimble RM. Pediatric Burns. In: International Encyclopedia of Rehabilitation. (2010), Available at: http://cirrie.buffalo.edu/encyclopedia / en/article/119/ 